\title{
Calidad ecotoxicológica de los sedimentos en fiordos del sur de Chile
}

\author{
Ecotoxicological quality of sediments in fiords of southern Chile
}

\author{
Anny Rudolph ${ }^{1}$, Paulina Medina ${ }^{1}$, Ramón Ahumada ${ }^{1}$ y Vanessa Novoa ${ }^{1}$ \\ ${ }^{1}$ Departamento de Química Ambiental, Facultad de Ciencias, Universidad Católica de la Santísima Concepción, Casilla 297, \\ Concepción, Chile. annyr@ucsc.cl
}

\begin{abstract}
We analysed the ecotoxicological quality of sediments in Moraleda, Puyuguapi and Elefantes Channels and Aysén Fjord, located between $44^{\circ}$ and 46.5 으, using non-specific tests in order to evaluate their base conditions in terms of possible pollutants that may have been introduced in the area. The survival tests with Ampelisca araucana and Tisbe Iongicornis and fertility test with Arbacia spatuligera indicate that the sediments do not present problems of toxicity. Nevertheless, the increased cellular density observed in the tests with microalgae (Dunaliella tertiolecta, Dunaliella salina and Isochrysis galbana) indicates the accumulation of sediments rich in nutrients within the area.
\end{abstract}

Key words: Arbacia spatuligera, Ampelisca araucana, Tisbe Iongicornis, microalgae

\section{INTRODUCCIÓN}

La zona de fiordos y canales del Sur de Chile, por su particular geografía se ha visto enfrentada en las últimas décadas a estrés ambiental, debido a la explotación de sus recursos naturales (pesquerías y silvicultura), crecimiento de centros urbanos e industriales y actividades turísticos. Su columna de agua presenta una estructura de dos capas, una superficial de mayor variabilidad y una profunda homogénea, separadas por un gradiente vertical (ValleLevinson et al. 2002, 2007). La capa superficial presenta aguas cálidas, menos salinas, con alto contenido de oxígeno disuelto (> $120 \%$ de saturación), alta clorofila $a$ y baja concentración de nutrientes (Silva et al. 1997, Guzmán \& Silva 2002). El contenido menor de oxígeno disuelto puede alcanzar valores de $2,5 \mathrm{ml} \mathrm{L}^{-1}$ en la cabeza los fiordos Puyuguapi y Aysén, no registrándose zonas anóxicas (Silva et al. 1997, Silva \& Guzmán 2006).

La región presenta elevadas tasas de precipitación y bajas temperaturas, condiciones que favorecen una potencial transferencia de contaminantes desde la atmósfera a la zona costera y a los cuerpos de agua y sedimentos (Wania \& Mackay 1996). La entrada adicional de sustancias químicas a la columna de agua, proveniente de la actividad humana queda registrada en los sedimentos a través de fracciones de los contaminantes que reacciona con el material particulado o coloidal y que es atrapada en sitios sedimentarios (Pineda 2009). El conocimiento y vigilancia de un ecosistema permite, mediante la información recopilada, estar en condiciones de predecir a través de la implementación de modelos, los cambios ambientales que pueden ocurrir a futuro (Sánchez-Bayo 2009, Choueri et al. 2010).

Los estudios de toxicidad han demostrado que las distintas especies de organismos presentan un amplio rango de sensibilidades a una extensa diversidad de los contaminantes ambientales, lo que hace recomendable utilizar una batería de especies distintas para evaluar la toxicidad. Para que las pruebas puedan constituirse en una técnica de evaluación aceptada, de los organismos seleccionados, debe conocerse su biología, factibilidad de mantenerse 'in vitro', presentar alta sensibilidad a los tóxicos y ser factible la reproducibilidad de los ensayos (Silva et al. 2007).

El presente estudio analiza a través de bioensayos no específicos la calidad ecotoxicológica de los sedimentos en los canales Moraleda, Puyuguapi, Golfo Elefantes y Fiordo Aysén, ubicados entre los $44^{\circ}$ y $46,5^{\circ} \mathrm{S}$, sur de Chile, con el objeto de evaluar, utilizando especies blanco, su condición toxicológica en relación a posibles contaminantes biodisponibles, introducidos o generados en el área. Para cumplir con este objetivo se seleccionaron como indicadores de la calidad del sedimento, pruebas de supervivencia con juveniles del anfípodo epibentónico Ampelisca araucana (Gallardo, 1962) y copépodo harpacticoide Tisbe longicornis (George, 1993), pruebas de fecundación con gametos del equinodermo Arbacia spatuligera (Valenciennes, 1841) y de densidad celular (cél ml-1) con la microalgas Isochrysis 
galbana (Parke), Dunaliella tertiolecta (Butcher, 1959) y Dunaliella salina (Dunal). La información resultante puede ser utilizada con diversos fines, e.g., comparación, regulación e implementación de modelos en la predicción de posibles alteraciones sobre el ambiente estudiado.

\section{Materiales Y MÉTODOS}

Las muestras de sedimentos se obtuvieron siguiendo el eje central de los cuerpos de agua, en noviembre de 2007, durante la Campaña CIMAR 13 Fiordo, desde el buque 'AGOR Vidal Gormaz' utilizando un box corer de $0,08 \mathrm{~m}^{3}$. Se recolectaron aproximadamente $250 \mathrm{~g}$ de los primeros 5 $\mathrm{cm}$ de sedimentos, obteniéndose 2 muestras en canal Moraleda, 3 en el canal Puyuguapi, 2 en Golfo Elefantes y 6 en el Fiordo Aysén (Fig.1). Cada muestra fue obtenida en triplicado y colocadas en bolsas plásticas, que fueron guardadas en envases de polietileno rotulados y congelados a $-18^{\circ} \mathrm{C}$. Dado lo lejano del sitio de muestreo y el extenso periodo del crucero, los análisis se realizaron entre 30 y 80 días después de su recolecta y arribo del crucero.
Las especies Tisbe longicornis, Ampelisca araucana y Arbacia spatuligera fueron recolectadas en un área no alterada en la Bahía Coliumo 3650’'S, 7255’W (FuentesRíos et al. 2005, Altamirano-Chovar et al. 2006) y cultivadas en condiciones de laboratorio (acuarios de vidrio, aireación constante y alimentación en base a microalgas, Dunaliella tertiolecta, Dunaliella salina e Isochrysis galbana adquiridas en el 'Laboratorio de Cultivos' de la Universidad de Concepción.

En las pruebas de toxicidad se trabajó exponiendo directamente a los organismos con los sedimentos o a un lixiviado de ellos. El lixiviado de cada muestra se realizó según la metodología de Dinnel \& Strober (1985), agitando 50 g de sedimento con $50 \mathrm{~mL}$ de agua de mar filtrada y aireada, en un Heidolph Unimax ${ }^{\circledR} 2010$ a 5 rpm por 10 min, para posteriormente dejar en frío $\left(4^{\circ} \mathrm{C}\right)$ por $12 \mathrm{~h}$, separando la fase líquida (lixiviado) de los sedimentos. Los controles en cada ensayo fueron realizados según las sugerencias de cada metodología.

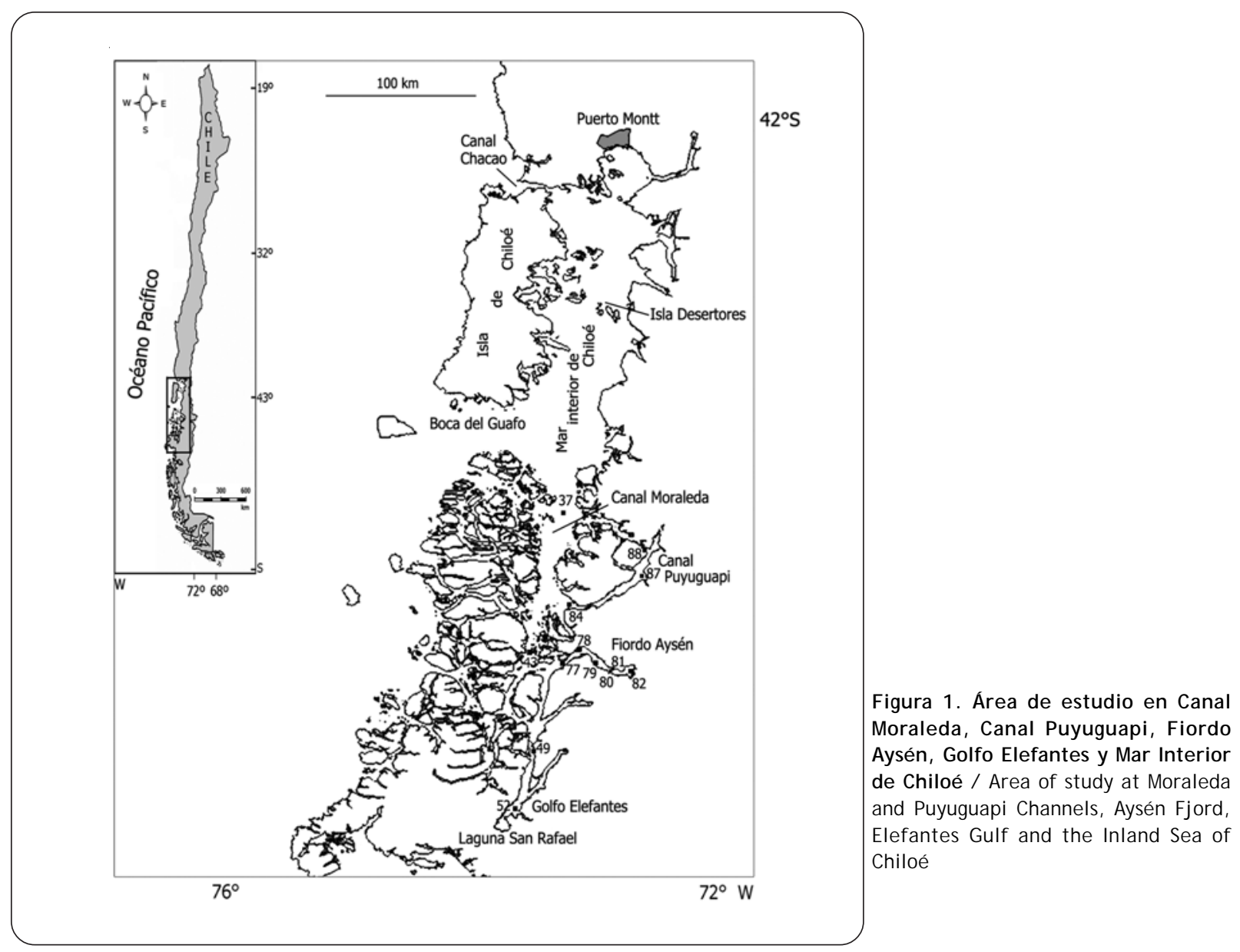




\section{Prueba de Supervivencia con Ampelisca araucana}

El ensayo se condujo siguiendo las sugerencias de Soto et al. (2000), esto es, los organismos fueron recolectados desde los sedimentos marinos con un tamiz de $500 \mu \mathrm{m}$. En el laboratorio se seleccionaron organismos de ca. $6 \mathrm{~mm}$ de longitud los que fueron mantenidos con aireación constante a $13^{\circ} \mathrm{C}$ y alimentación en base a microalgas durante su aclimatación. Para el ensayo se utilizaron cubetas con 200 g de sedimentos y $300 \mathrm{~mL}$ de agua de mar filtrada, con 5 individuos por cubeta y en triplicado; por un periodo de 10 días, sin aireación ni alimentación. Los resultados fueron contrastados contra controles negativos, preparados con sedimentos del lugar de extracción de los organismos. Los ensayos de sensibilidad (control positivo) se condujeron con soluciones de $\mathrm{K}_{2} \mathrm{Cr}_{2} \mathrm{O}_{7}$ (p.a) entre 0 y $100 \mathrm{mgL}^{-1}$ por un periodo de 4 días.

\section{Prueba de supervivencia con Tisbe longicornis}

El ensayo se realizó siguiendo la metodología propuesta por Larraín et al. (1998a); esto es, se seleccionó hembras ovígeras adultas con un tamiz de $250 \mu \mathrm{m}$, y luego fueron mantenidas en acuarios de vidrio, con aireación constante y alimentación en base a microalgas, hasta la eclosión de las larvas. El ensayo consideró organismos juveniles de una misma cohorte de ca. 15 días de vida (i.e., 0,6 mm de longitud). Se utilizó lixiviado preparado con el sedimento bajo prueba. Los resultados se contrastaron con controles negativos. Para las pruebas de sensibilidad se utilizó soluciones de $\mathrm{CuSO}_{4(\mathrm{p} \text {.a) }}$ entre 1 y $100 \mu \mathrm{gL}^{-1}$. La duración de los ensayos fue de $48 \mathrm{~h}$ en forma paralela, con 5 individuos por cubeta y en triplicado.

\section{Prueba de fecundación con Arbacia spatuligera}

El ensayo se realizó siguiendo la metodología propuesta por Zúñiga (1999). Básicamente consistió en producir una fecundación artificial de los óvulos del erizo A. spatuligera, en presencia de un lixiviado preparado con el sedimento bajo prueba. Los resultados se contrastaron con sus controles negativos. Para el análisis de sensibilidad se utilizó soluciones de $\mathrm{CuSO}_{4}$ (p.a) entre 0 y $50 \mu \mathrm{gL}^{-1}$. Los ensayos fueron realizados por un periodo de $60 \mathrm{~min}$, utilizando soluciones de 7 × $10^{7}$ espermios $\mathrm{mL}^{-1}$ y 2.000 óvulos $\mathrm{mL}^{-1}$ en cuadruplicado.

\section{Prueba de DENSIDAd CELUlar Con Microalgas}

Estas se realizaron según las sugerencias metodológicas propuestas por USEPA (1988), Cifuentes et al. (1998) y la NCh 2706 (2002) ${ }^{1}$. Las microalgas fueron cultivadas con el medio de cultivo Guillar a $17^{\circ} \mathrm{C} \pm 1$ y luz PAR continua de 28.000 lux. Se utilizó $10 \mathrm{~mL}$ del lixiviado preparado con el sedimento bajo prueba e inóculos de cultivo inicial. Los resultados se contrastaron con controles negativos. El análisis de sensibilidad se realizó con concentraciones de $\mathrm{K}_{2} \mathrm{Cr}_{2} \mathrm{O}_{7}$ (p.a) entre 0 y $100 \mathrm{mgL}^{-1}$. Los ensayos se realizaron en forma paralela y en cuadruplicado. La densidad celular (cél ml-1) fue determinada mediante recuento de células con cámara de Neubauer a tiempo cero y a 96 h.

\section{AnÁLISIS ESTAdísticos}

Los diferentes índices de los ensayos de sensibilidad i.e., $\mathrm{CL}_{50}$ (concentración letal para el 50\% de los organismos sometidos al ensayo) para Ampelisca araucana y Tisbe longicornis; $\mathrm{CE}_{50}$ (concentración efectiva en el 50\% de los organismos sometidos al ensayo) en el ensayo de Arbacia spatuligera y el $\mathrm{CI}_{50}$ [concentración que inhibió al 50\% el crecimiento celular (cél $\mathrm{mL}^{-1}$ ) de la microalga] en Dunaliella tertiolecta, Dunaliella salina e Isochrysis galbana, fueron calculados mediante el análisis probit (EPA Probit Analysis Program, versión 1,4) (Finney 1971). Para analizar la normalidad de los datos se aplicó el test de Shapiro-Wilk y para observar la homogeneidad de la varianza se utilizó el test de Cochrane. Los porcentajes de supervivencia, fecundación y densidad celular de cada especie fueron comparados con su respectivo grupo control, a través de una prueba paramétrica ANDEVA de una vía. En el caso de encontrar diferencias significativas, se aplicó una prueba $a$ posteriori de Tukey. Los resultados del aumento de densidad celular en el ensayo con I. galbana no arrojaron una distribución normal por lo que se aplicó una prueba no paramétrica de Kruskall-Wallis; las diferencias significativas fueron analizadas a posteriori a través de comparaciones múltiples de los valores de $P$ de las muestras y del control. Todos los análisis estadísticos se realizaron a través del programa computacional STATISTICA versión 6.0 (StatSoft. Inc. 2001) ${ }^{2}$.

${ }^{1} \mathrm{NCh}$ 2706. 2002. Calidad de agua - bioensayo de inhibición de crecimiento de algas en agua dulce con Selenastrum capricornutum (Raphidocelis subcapitata). Instituto Chileno de Normalización, INN, Santiago de Chile, 28 pp.

${ }^{2}$ StatSoft. Inc. (2001). Statistica (Data Analysis Software System). <www. statsoft.com>. 


\section{RESULTADOS Y DISCUSIÓN}

La sensibilidad de los organismos blanco utilizados en este estudio, frente a los tóxicos utilizados i.e., dicromato de potasio o sulfato de cobre (control positivo), fue semejante a los informados, lo que los hace resultados factibles de comparar respecto de la calidad del sedimento. En los ensayos con Ampelisca araucana frente a soluciones de dicromato de potasio el $\mathrm{CL}_{50}$ obtenido fue de 55,37 $\mathrm{mgL}^{-1}$ semejante a lo informado por Larraín et al. (1998b) y Soto et al. (2000); en los ensayos con Tisbe longicornis el $\mathrm{CL}_{50}$ estimado fue de $56,92 \mu \mathrm{gL}^{-1}$ para sulfato de cobre, semejante a lo informado por Larraín et al. (1998a); para Arbacia spatuligera la $\mathrm{CE}_{50}$ fue de $16,51 \mu \mathrm{gL}^{-1}$ de sulfato de cobre semejante a los informados por Aguirre-Martínez et al. (2009) y Rudolph et al. (2010). Los ensayos con microalgas i.e., Isochrysis galbana, Dunaliella tertiolecta y D. salina, mostraron IC $_{50}$ de 40; 65,47 y 66,14 mgL $^{-1}$ frente a soluciones de dicromato de potasio respectivamente, sensibilidades semejantes a los informados en Rudolph et al. (2010).

Los sedimentos en las localidades analizadas no evidenciaron problemas de toxicidad, ya que se observaron supervivencia promedio del $100 \%$ en las especies blanco i.e., Ampelisca araucana y Tisbe longicornis, sin diferencias significativas respecto de los controles $\left(\mathrm{F}_{(13,28)}=0,863 ; P=\right.$ 0,596 y $_{(13,28)}=0,92 ; P=0,54$, respectivamente).

La prueba de fecundación con gametos de Arbacia spatuligera mostró diferencias significativas en los porcentajes de fecundación con el grupo control $\left(\mathrm{F}_{(13,28)}=\right.$ $7,3, P<0,05$ ) en las muestras 52 (con un $97 \%$ ) y 80 (con un $97,5 \%$ ). No obstante, los porcentajes medidos superan el $95 \%$ de fecundación, lo que acuerdo a Aguirre-Martínez et al. (2009), para pruebas de toxicidad con lixiviado del sedimento, puede considerarse semejante al comportamiento del control.

En los ensayos con microalgas, Isochrysis galbana mostró un crecimiento significativo respecto del control en el $69 \%$ de las muestras $\left(\mathrm{H}_{(13,42)}=29,34, P=0,0058\right)$ i.e., 49, 52, 77, 78, 79, 80, 81 y 84 (Fig. 2). En los ensayos con Dunaliella salina, se observó diferencias significativas sólo en el $39 \%$ de las muestras respecto del control $\left(\mathrm{F}_{(13,28}\right)=$ $14,53, P<0,01$ ) en las muestras $78,81,82,87$ y 88 (Fig. 3 ) y en los ensayos con Dunaliella tertiolecta se observó una situación similar, donde igual número de muestras registró una mayor densidad celular respecto del control $\left(\mathrm{F}_{(13,28)}=\right.$ 2,41, $P=0,025)$, i.e., 78, 81, 82, 87 y 88.

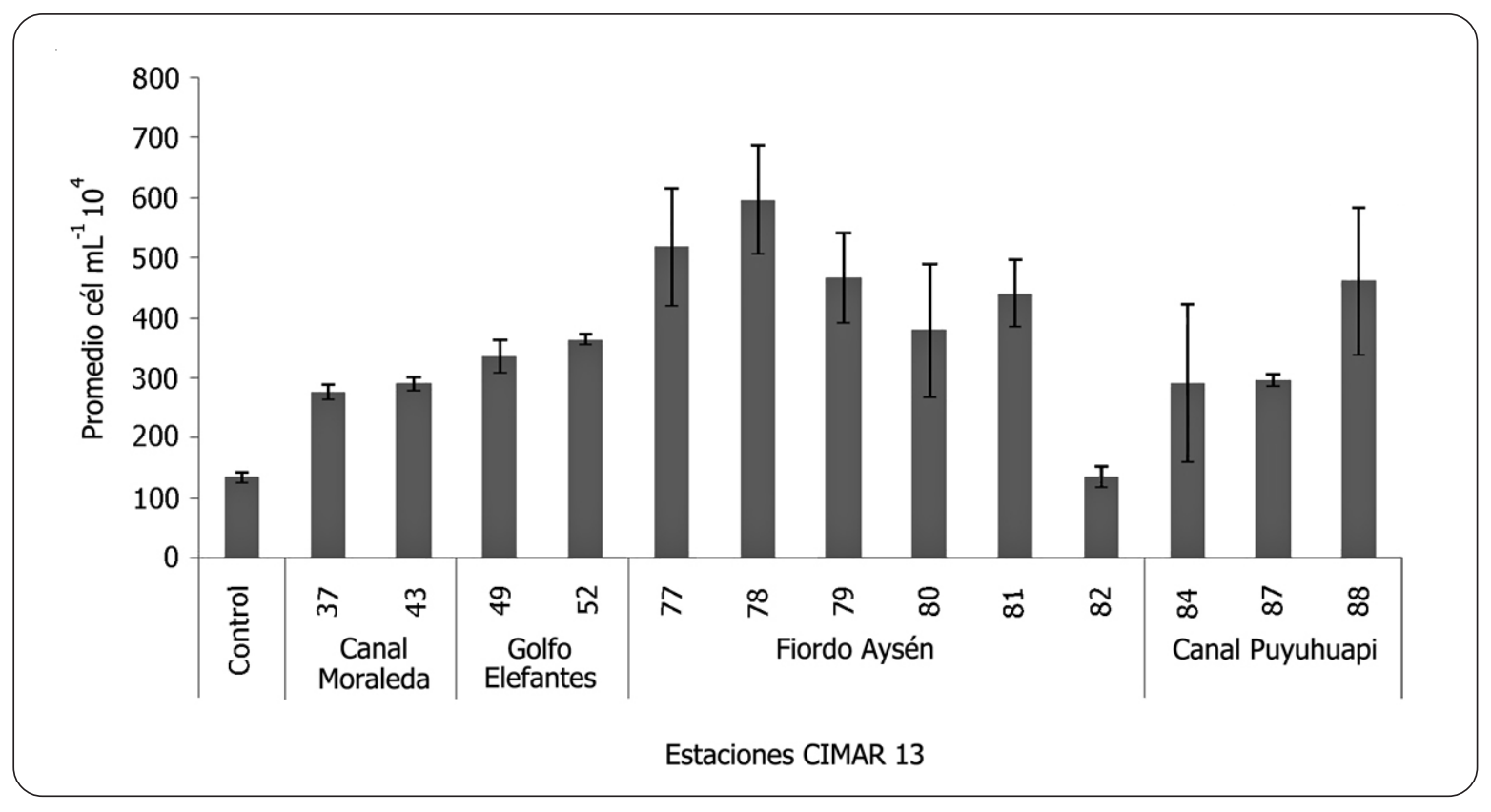

Figura 2. Porcentaje de crecimiento promedio de Isochrysis galbana en elutriados de sedimentos y controles. Campaña CIMAR 13, noviembre 2007. Línea sobre cada barra muestra la desviación estándar / Average percentage of growth for Isochrysis galbana in elutriates of sediments and controls. CIMAR 13, November 2007. Line on bar shows the standard deviation 
El crecimiento observado en los ensayos, puede ser atribuido a la presencia de nutrientes en los lixiviados, los cuales se habrían liberado durante su preparación desde los sedimentos. Estos nutrientes habrían estimulado el crecimiento de las microalgas, sumado a la ausencia de tóxicos, lo que se confirma al observar que ningún cultivo presentó menor densidad celular que los controles. La liberación de nutrientes se atribuye a cambios locales, que podrían estar generando un desbalance en el contenido de fósforo y/o nitrógeno.

Una condición semejante, con incrementos en la densidad celular de las muestras respecto de los controles, se observó en los ensayos realizados con sedimentos recolectados en el sector central en fiordos y canales del Mar Interior de Chiloé, durante la Campaña CIMAR 10 Fiordos (Rudolph et al. 2007) y la Campaña CIMAR 11 Fiordos (Rudolph et al. 2009). En cambio, una situación diferente se observó con las muestras de sedimentos recolectadas en sectores costeros cercanos a centros de cultivo en el Mar Interior de Chiloé, sedimentos en su mayoría anóxicos, en que además de no observarse crecimento de los ensayos con microalgas se observó toxicidad (Rudolph et al. 2009).

La calidad ecotoxicológica de los sedimentos estudiados mediante las prueba de fecundación con Arbacia spatuligera, al igual que las pruebas de supervivencia con Ampelisca araucana y Tisbe longicornis, indican que en el sedimento de las localidades estudiadas no se detecta la presencia de tóxicos biodisponibles. Los ensayos con microalgas no evidencian problemas de toxicidad e incrementaron su crecimiento, sugiriendo la acumulación de sedimentos ricos en nutrientes. Esto último, estaría indicando el paso de un sistema oligotrófico a un sistema mesotrófico a futuro. Las condiciones informadas para el área, i.e., saturación de oxígeno en la capa superficial y la ausencia de anoxia en la capa profunda (Silva et al. 1997, Guzmán \& Silva 2002, Silva \& Guzmán 2006), confirman que las localidades estudiadas, se comportan aún como un sistema poco alterado.

\section{Agradecimientos}

Los autores agradecen a la Dirección de Investigación de la UCSC; al Comité Oceanográfico Nacional por el financiamiento de esta investigación a través del Proyecto CONA 13F 07-03. A la Srta. Emma Cascales (UCSC) por la toma de muestras durante el Crucero y al Prof. Pablo Venegas (Laboratorio de Acuicultura, UCSC) por permitirnos trabajar en su laboratorio con condiciones reguladas de temperatura.

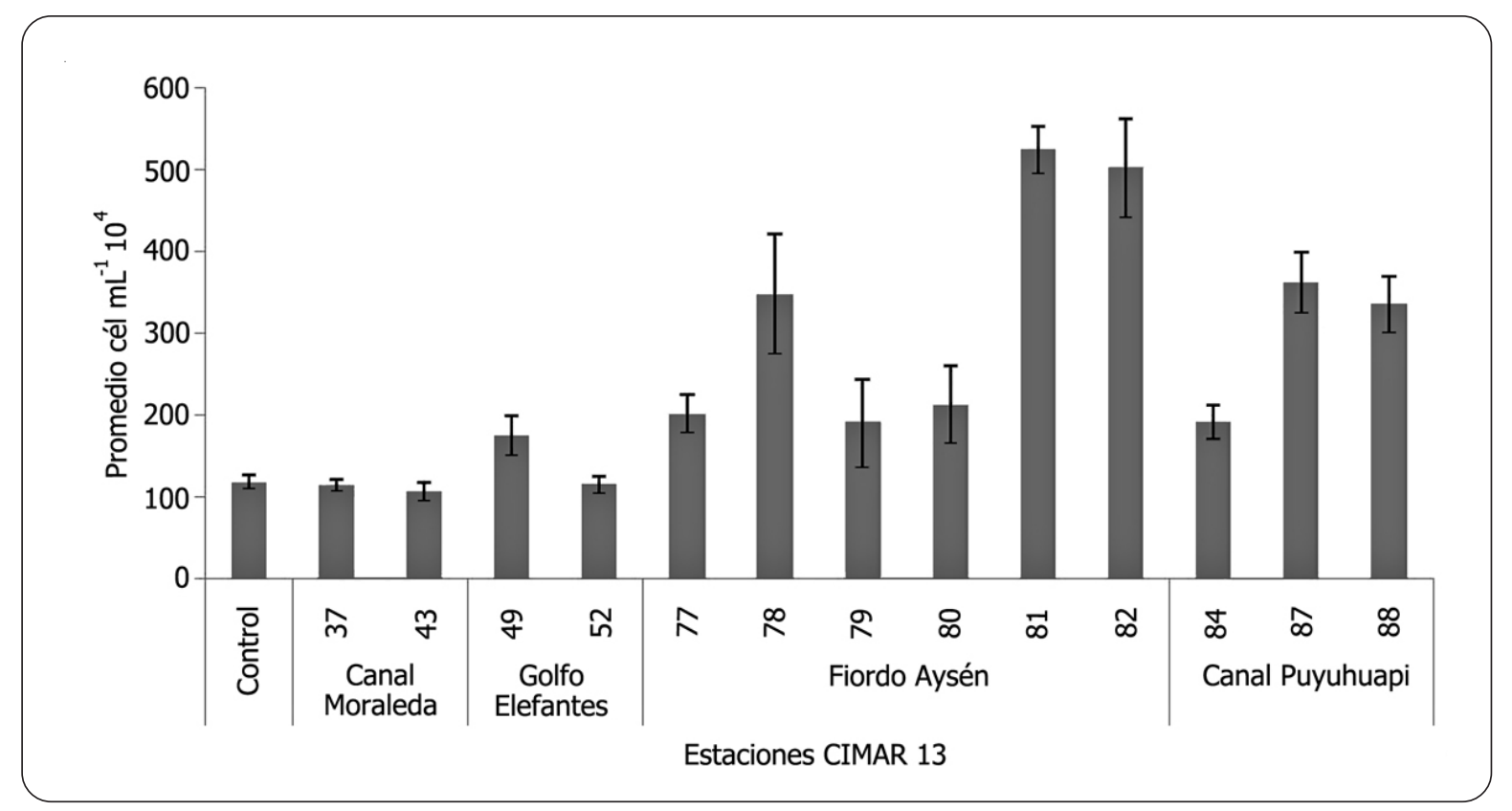

Figura 3. Porcentaje de crecimiento promedio de Dunaliella salina en elutriados de sedimentos y controles. Campaña CIMAR 13, noviembre 2007. Línea sobre cada barra muestra la desviación estándar / Average percentage of growth for Dunaliella salina in elutriates of sediments and control. CIMAR 13, November 2007. Line on each bar shows the standard deviation 


\section{LITERATURA CITADA}

Aguirre-Martínez G, A Rudolph, R Ahumada, R Loyola \& V Medina. 2009. Toxicidad no específica en sedimentos portuarios, una aproximación al contenido de contaminantes críticos. Revista de Biología Marina y Oceanografía 44(3): 725-735.

Altamirano-Chovar C, A Rudolph \& R Sepúlveda. 2006. Diferential sensitivity to varying degrees of human influence in juvenile Semimytilus algosus (Gould, 1950) (Mollusca: Mytilidae): From four coastal sites in southcentral Chile. Bulletin of Environmental Contamination Toxicology 77: 171-178.

Choueri RB, A Cesar, DMS Abessa, RJ Torres, I Riba, CDS Pereira, MRL Nascimento, RD Morais, AA Mozeto \& TA del Valls. 2010. Harmonised framework for ecological risk assessment of sediments from ports and estuarine zones of North and South Atlantic. Ecotoxicology 19(4): 593-825.

Cifuentes A, J Silva, E Bay-Schmith \& A Larrain. 1998. Selección de cepas de microalgas para ser utilizadas en bioensayos de toxicidad. Gayana Oceanológica 61(1-2): 1-9.

Dinnel PA \& QJ Strober. 1985. Methodology and analysis of sea urchin embryo bioassays. Fisheries Research Institute. University of Washington, Seattle, Circular 85: 1-319.

Finney DJ. 1971. Probit analysis, 333 pp. Cambridge University Press, Cambridge.

Fuentes-Ríos D, R Orrego, A Rudolph, G Mendoza, JF Gavilán \& R Barra. 2005. EROD activity and biliary fluorescence in Schroederichthys chilensis (Guichenot 1848): Biomarkers of PAH exposure in coastal environments of the Pacific Ocean. Chemosphere 61: 192199.

Guzmán D \& N Silva. 2002. Caracterización física y química y masas de agua en los canales australes de Chile entre Boca del Guafo y Golfo Elefantes (Crucero CIMAR Fiordo 4). Ciencia y Tecnología del Mar 25(2): 45-76.

Larraín A, E Soto, J Silva \& E Bay-Schmith. 1998a. Sensibility of meiofaunal copepod Tisbe longicornis to $\mathrm{K}_{2} \mathrm{Cr}_{2} \mathrm{O}_{7}$ under varying temperature regimes. Bulletin of Environmental Contamination and Toxicology 61: 391-396

Larraín A, E Soto \& E Bay-Schmith. 1998b. Assessment of sediment in San Vicente Bay, Central Chile, using the amphipod Ampellisca araucana. Bulletin of Environmental Contamination and Toxicology 61: 363-369.

Pineda V. 2009. Granulometría y geoquímica de los sedimentos marinos en el área comprendida entre el Seno Reloncaví y Golfo Corcovado, Chile. Ciencia y Tecnología del Mar 32(1): 27-47.

Rudolph A, G Aguirre, J Moscoso, N Silva \& R Ahumada. 2007. Sediment quality between Reloncaví Gulf and
Corcovado Gulf $\left(41.5-43^{\circ} \mathrm{S}\right)$ based on toxicity tests. Investigaciones Marinas 35(2): 53-61.

Rudolph A, P Medina, C Urrutia \& R Ahumada. 2009. Ecotoxicological sediment evaluations in marine aquaculture areas of Chile. Environmental Monitoring and Assessment 155: 419-429.

Rudolph A, P Medina, V Novoa, R Ahumada \& I Cortés. 2010. Calidad ecotoxicológica de sedimentos en sectores del Mar Interior de Chiloé, Campaña CIMAR 12 Fiordos. Ciencia y Tecnología del Mar 33(1): 17-29.

Sánchez-Bayo F. 2009. From simple toxicological models to prediction of toxic effects in time. Ecotoxicology 18: 343354.

Silva N \& D Guzmán. 2006. Condiciones oceanográficas, físicas y químicas, entre boca del Guafo y fiordo Aysén (Crucero Cimar 7 Fiordos). Ciencia y Tecnología del Mar 29(1): 25-44.

Silva N, C Calvete \& H Sievers. 1997. Características oceanográficas físicas y químicas de canales australes chilenos entre Puerto Montt y laguna San Rafael. Ciencia y Tecnología del Mar 20: 23-106.

Silva J, C Fuentealba, E Bay-Schmith \& A Larrain. 2007. Estandarización del bioensayo de toxicidad aguada con Diplodon chilensis usando un tóxico de referencia. Gayana 71(2): 135-141.

Soto E, E Larrain \& E Bay-Schmith. 2000. Sensitivity of Ampelisca araucana juveniles (Crustacea: Amphipoda) to organic and inorganic toxicants in test of acute toxicity. Bulletin of Environmental Contamination and Toxicology 64: 574-578.

USEPA. 1988. Short-term methods for estimating the chronic toxicity of effluents and receiving waters to marine and estuarine organisms. Office of Research and Development, U.S. Environmental Protection Agency, Washington D.C. 206460. EPA/600/4-87-028. [en línea] < http:// www.epa.gov/eerd/westmethman.htm>

Valle-Levinson A, M Cáceres, HH Sepúlveda \& K Holderied. 2002. Flow patterns in the channels associated to the mouth of Aysén sound. Ciencia y Tecnología del Mar 25: 5-16.

Valle-Levinson A, N Sarkar, R Sanay, D Soto \& J León. 2007. Spatial structure of hydrography and flow in a Chilean fjord, estuario Reloncavi. Estuaries and Coasts 30(1): 113-126.

Wania F \& D Mackay. 1996. Tracking the distribution of persistent organic pollutants. Environmental Science \& Technology 30: 390-396.

Zúñiga M. 1999. Evaluación de la calidad acuática de bahía San Jorge a través de ensayos de toxicidad crónica con gametos del erizo de mar Arbacia spatuligera. Ciencia y Tecnología del Mar 22: 59-74.

Recibido el 27 de mayo de 2010 y aceptado el 28 de octubre de 2010 